\title{
The Role of Genetics in Delirium in the Elderly Patient
}

\author{
Barbara C. van Munster ${ }^{a, b}$ Sophia E. de Rooij ${ }^{b}$ Johanna C. Korevaar ${ }^{a}$ \\ a Department of Clinical Epidemiology, Biostatistics and Bioinformatics, and bepartment of Internal Medicine, \\ Academic Medical Centre, Amsterdam, The Netherlands
}

\section{Key Words}

Delirium, diagnosis · Genetics • Risk factors, delirium •

Pathophysiology $\cdot$ Review

\begin{abstract}
Delirium is an important neuropsychiatric syndrome in the elderly that is associated with an increase in mortality, impaired physical and cognitive recovery, and increased healthcare costs. Two important gaps in daily practice with delirium are the pathophysiological obscurity and the low recognition rates. Genetics offers the possibility to contribute knowledge to both of these gaps with its unique and diverse techniques. However, genetic studies in delirium have been scarce until recently, due to the nature of the diagnosis, the etiology of the syndrome, and the pitfalls inherent in the design of genetic studies. This review describes the barriers of this field of research and the implications for our current knowledge of delirium.
\end{abstract}

Copyright $\odot 2009$ S. Karger AG, Basel

\section{Introduction}

Delirium is an important neuropsychiatric syndrome with frequencies in the elderly population of up to $56 \%$ at and during admission [1]. Though patients usually recover after treatment of the precipitating factors, delirium is independently associated with an increase in mortality, impaired physical and cognitive recovery, and increased healthcare costs [2]. Since the proportion of elderly people with greater risk for delirium continues to grow, the absolute number of patients with delirium and associated problems can be expected to rise in the future.

Despite its large impact, the pathophysiological mechanisms leading to delirium are still largely unknown [3]. Furthermore, the delirium recognition rates are low (12-43\%), and its management remains consequently inadequate in up to $80 \%$ of the patients [1]. Genetics, the science of heredity and variation, has been named as the most promising technique to contribute to both of these gaps $[4,5]$. Despite the fact that they offer unique possibilities to learn more about the pathophysiology of delirium, studies on genetics in delirium in elderly patients have been scarce until now. The field of genetics has already acquired a prominent place in pathophysiological research in other psychiatric disorders like dementia, schizophrenia and depression [6-8]. In these disorders, its value has been demonstrated due to both common variations of the human genome that are becoming available as well as new high-throughput technologies [9].

The scarcity of genetic studies in delirium may be due to the characteristics and etiology of the syndrome; genetic association studies in delirium are less straightforward than genetic association studies in other diseases of

\section{KARGER \\ Fax +4161306 1234 E-Mail karger@karger.ch} www.karger.com
(C) 2009 S. Karger AG, Basel

$1420-8008 / 09 / 0283-0187 \$ 26.00 / 0$

Accessible online at:

www.karger.com/dem
B.C. van Munster

Department of Clinical Epidemiology, Biostatistics and Bioinformatics

Room J1B-207-1, Academic Medical Centre, University of Amsterdam, PO Box 22660

NL-1100 DD Amsterdam (The Netherlands)

Tel. +31 20566 6945, Fax +31 20691 2683,E-Mail b.c.vanmunster@amc.uva.nl 
old age. This review will discuss this topic in detail. Additionally, we describe how genetics may contribute to knowledge regarding the pathophysiology of delirium and its implications for future research.

\section{The Syndrome of Delirium}

Delirium is defined by a fluctuating alteration in consciousness and a change in cognition or a perceptual disturbance [10]. It is evoked by a somatic factor, which can involve a variety of different illnesses, surgery, or substance (medication) intoxication or withdrawal. The different clinical subtypes of delirium include the hyperactive, hypoactive and mixed types. The diagnosis has to be made by an experienced clinician based on a classification scale like the Diagnostic and Statistical Manual of Mental Disorders [10] or the International Classification of Diseases [11]. Predisposing factors include higher age and cognitive and functional impairment, among others [12]. In addition to cognitive impairment serving as an important risk factor for patients developing delirium, delirious patients are also more prone to exhibit deterioration in their cognitive functioning afterwards [13].

The diagnosis is frequently missed due to the manifestation of the syndrome [1]. First, symptoms develop acutely, and the duration of delirium is limited to a few days if the precipitating factor is adequately treated. Therefore, the time frame for making an adequate diagnosis is short [14]. Second, symptoms of delirium fluctuate over time; because they are the most apparent during the night, the patient has to be closely observed for $24 \mathrm{~h}$. Finally, the hypoactive subtype, with its absence of overt distress or disturbance, is especially likely to be overlooked [15]. Apart from failure to recognize the symptoms, misdiagnosis is a considerable problem, since depression and dementia are important differential diagnoses for hypoactive delirium. The hyperactive and mixed subtypes of delirium may falsely be diagnosed as functional psychosis, dementia, (hypo)mania, anxiety disorders or akathisia [16].

\section{The Pathophysiology of Delirium}

A number of hypotheses have been put forward in an attempt to explain the pathophysiological processes leading to the development of delirium $[3,5,17,18]$. Several theories describe disturbances of different systems in the brain, such as the (1) 'neurotransmitter', (2) 'inflammatory', (3) 'physiological stress', (4) 'cellular signaling', (5) 'oxygen supply' or (6) 'sleep-wake cycle' systems. The burden of proof for the diverse hypotheses varies from almost hypothetical (the role of melatonin) to fairly proven (dysbalance between dopamine and acetylcholine activity in the brain).

The most widely propagated theory focuses on the neurotransmitter system. This theory states that relative acetylcholine deficiency and dopamine excess could mediate the characteristic symptoms of delirium [17]. This is supported on the one hand by the fact that delirium can be evoked by dopamine agonists and anticholinergic medication and on the other hand because delirium can be successfully treated with dopamine receptor antagonists and possibly also by cholinesterase inhibitors [19].

Proinflammatory cytokines are known to contribute to the development of sickness behavior. This syndrome is characterized by symptoms overlapping with delirium and can be induced by a wide variety of clinical conditions just like delirium [20]. Recently, an association between the proinflammatory cytokines interleukin- 6 and interleukin-8 and delirium was found in medical [21] and surgical [22] patients.

There is some evidence that dysregulation of the limbic-hypothalamic-pituitary-adrenal axis, with pathologically sustained high levels of cortisol occurring with acute stress, can precipitate and/or sustain delirium [3].

The 'cellular signaling hypothesis' suggests that more fundamental processes like intraneuronal signal transduction may be disturbed, thereby affecting neurotransmitter synthesis and release [18].

The 'oxygen deprivation hypothesis' proposes that decreased oxidative metabolism in the brain causes cerebral dysfunction because of abnormalities in various neurotransmitter systems [18].

Disruption of the sleep-wake cycle is an important characteristic of delirium. Melatonin, a hormone involved in the circadian rhythm, could be responsible for the disturbance in this system [23]. Several studies have shown a difference in melatonin secretion in patients with delirium compared to patients without delirium [23, 24].

Since many of the above systems interact, these theories are probably not mutually exclusive. On the contrary, since the syndrome of delirium is the result of a wide variety of combinations of predisposing and precipitating factors, the concept of a final common pathway seems to be the most plausible [17]. The candidate system for this final common pathway that is most widely supported by the existing evidence is the neurotransmitter system. 


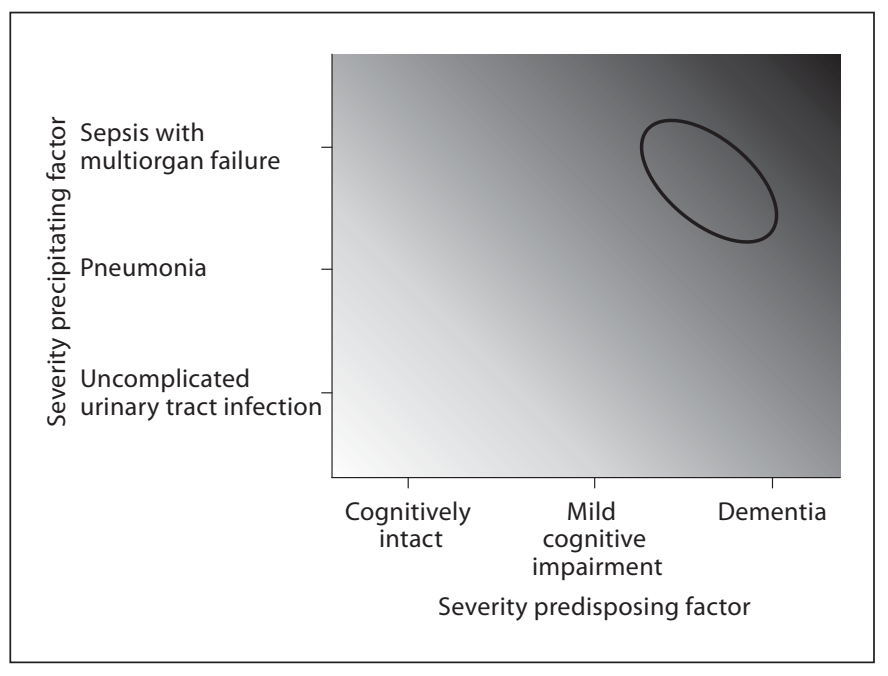

Fig. 1. Relationship between predisposing and precipitating factors. Darker shading indicates a higher risk for delirium. Genetic factors will play the largest role in the delirium phenotype in the light zone. In analogy, the contribution of genetics to the nondelirium phenotype will be largest in the darker zone.

\section{The Phenotype Delirium}

A phenotype is any observable characteristic of an organism, such as its morphology, development, biochemical or physiological properties, or behavior. Phenotypes result from the expression of an organism's genes, influence of environmental factors and possible interactions between the two. Experiencing delirium is also a phenotype. This delirium phenotype is by definition dependent on environmental factors; a patient needs to cross a certain threshold with a combination of predisposing and precipitating factors to elucidate it [25]. Figure 1 suggests a possible relationship between predisposing and precipitating factors in the risk for delirium. While a person with many predisposing factors will only need a minor trigger to develop delirium (i.e. an old demented patient with a mild urinary tract infection), a person without predisposing factors requires a very serious trigger (i.e. a young patient with severe sepsis in the intensive care unit). This figure reveals that it is difficult to clearly classify a person as demonstrating a nondelirious phenotype: if there are no precipitating factors, the phenotype of delirium is unknown. If there is a precipitating factor, however, the classification of the phenotype as delirious or not is strongly influenced by the severity of both the predisposing and precipitating factors. The classification of the nondelirium phenotype can thus only be made with certainty in patients without delirium despite severe predisposing and precipitating factors. In most other disorders, the people are more reliably classified as controls because these disorders have already revealed themselves at higher age. Moreover, misclassification of controls is not substantial if the frequency of the disorder is relatively low, whereas the frequency of delirium will become high with rising age.

In addition to the uncertainty in delirium phenotype classification, we do not know if there is only one delirium phenotype. In theory, it is possible that different etiologies may lead to delirium via different pathophysiological pathways. It could be possible, for example, that one patient will always experience delirium after surgery but never after infection. In the same respect, we do not know whether the hyperactive, hypoactive and mixed subtypes of delirium are all part of the same phenotype. Further, we do not know if the subtypes alternate during one episode of delirium [16].

\section{The Genotype Delirium}

The genotype of an organism is comprised of the inherited instructions it carries within its genes. Variation among individuals may be due to genetic and/or environmental factors. Heritability refers to the proportion of phenotypic variation in a population that is attributed to the genetic profile of the individual. Estimation of heritability is done with twin or family studies. The heritability of delirium is unknown, and there are no known families with a high frequency of affected members. However, that delirium might be heritable can be deduced from the estimated heritability of 30\% for the occurrence of any psychotic episode in late-onset Alzheimer's disease [26]. The most important reason accounting for the lack of heritability studies is the fact that delirium develops mainly in elderly people. In a family, therefore, the phenotype of younger members will be unknown, whereas older siblings have already passed away. This difficulty, in addition to the challenges in the proper classification of the phenotype described earlier, explains why twin and family studies in delirium are hard to perform.

Figure 1 shows the risk of delirium for different combinations of predisposing and precipitating factors. Predisposing factors vary and will grow over time; in addition to age, the incidence of other important factors predisposing to delirium (e.g. cognitive and functional impairment) rises with increasing age. Genetic features may also be part of these predisposing factors for deliri- 
um. Since genetic factors are relatively independent of age, the proportion of phenotypic variation that is attributed to genetics declines with increasing age. The diminished role of genetic factors in the etiology of disease at advanced age is not unique for delirium but has also been demonstrated in other old-age diseases like dementia and osteoporosis [27, 28].

We hypothesize that the contribution of genetics to the development of delirium will be largest in patients who become delirious despite minor predisposing and precipitating factors (lower left corner of figure 1). In analogy, the protective effect of genetic factors or the contribution of genetics to the nondelirium phenotype will be largest in patients without delirium despite the presence of major predisposing and precipitating factors (upper right corner in fig. 1). Both of these groups of patients represent ideal study populations for identifying genetic factors associated with delirium. The first group has the disadvantage of a low incidence of the delirium phenotype, whereas the second could have the drawback of a low incidence of the nondelirium phenotype. In an elderly hospitalized population (placed near the upper right corner in fig. 1), we would expect to identify more genetic factors that are protective rather than causative for delirium.

\section{Methods of Genetic Research in Delirium}

A candidate gene is a gene that is thought to be likely to cause a disease. The gene may be a candidate because it is located in a particular chromosome region suspected of being involved in the disease, or its protein product may suggest that it could be the disease gene in question. Although most substances involved in the pathophysiology are not proteins, many candidate genes can be derived from the different hypotheses of the pathophysiology of delirium. Table 1 shows the most obvious candidate genes for different theories, but even more genes could be considered as relevant [29]. Common and more complex diseases, like delirium, are usually not associated with a single gene but instead with a number of different genes [30]. If there are indeed more genes involved, we can expect each of them to have a relatively small effect. One commonly used technique that is sufficiently powerful to detect these small effects is the candidate gene approach.

Candidate gene association studies are relatively quick and inexpensive to perform. They can test the effect of genetic variants of a potential contributing gene (the candidate gene) in unrelated cases and controls. Most genes contain many known DNA sequence variations called single nucleotides polymorphisms (SNPs). The most interesting variations for association studies are functional SNPs, which can influence the trait of interest by producing proteins with an altered structure, function or concentration. These SNPs can be easily determined in DNA obtained from somatic cells, e.g. white blood cells.

While the candidate gene approach examines a single gene, genome-wide association studies simultaneously assay hundreds of thousands of SNPs using highthroughput genotyping technologies. Because the entire genome is analyzed for the genetic associations of a particular disease, this technique allows the genetics of a disease to be investigated in a non-hypothesis-driven manner. As multiple comparisons are being made, correction for multiple testing is required; this correction implies that large study populations are needed to provide sufficient power to detect significant associations.

Epigenetics refers to heritable changes in phenotype or gene expression caused by mechanisms other than changes in the underlying DNA sequence. Biological, chemical and physical factors as well as cultural and educational backgrounds influence gene functioning [30]. These gene-environment interactions may lead to epigenetic changes. For example, the methylation of DNA can affect genetic expression and thereby influence the risk of delirium. It will be important to delirium to determine both the genetic polymorphisms and epigenetic alterations in order to provide a comprehensive picture of its genetic etiology.

\section{Advantages of Genetic Research in Delirium}

Genetic research in relation to diseases offers two opportunities. The most important aim of genetic research into delirium is to learn more about the pathophysiology of the disease. Knowledge of the underlying processes is the starting point for the development of new medicines that have fewer adverse effects. Genetic research offers new possibilities in unraveling these mechanisms lacking in conventional research methods. First, conventional methods are hindered by the fact that delirium is a neuropsychiatric disorder: pathophysiological processes of interest can best be derived from brain research. Methods for brain research in humans are limited, since lumbar punctures and scans of the brain for research purposes are considered unethical in restless patients with an impaired capacity to provide informed consent. 
Table 1. Possible candidate genes deduced from the hypotheses of the pathophysiological mechanisms of delirium

\begin{tabular}{lll}
\hline Function & Candidate gene & Symbol \\
\hline Cytokine & Interleukin-6 & IL6 \\
Cytokine & Interleukin-6 receptor & IL6R \\
Cytokine & Interleukin-8 & IL8 \\
Acetylcholine & Acetylcholinesterase & ACHE \\
Cannabinoid & Cannabinoid receptor $1^{\text {a }}$ & CNR1 \\
Dopamine/norepinephrine & Catechol-O-methyltransferase & COMT \\
Dopamine & Dopamine receptor D1 & DRD1 \\
Dopamine & Dopamine receptor D2 & DRD2 \\
Dopamine & Dopamine receptor D3 & DRD3 \\
Dopamine & Solute carrier family 6, member 3 & SLC6A3 \\
Dopamine/norepinephrine & Tyrosine hydroxylase & TH \\
Glucocorticoid receptor & Nuclear receptor family 3, group C, member 1 & NR3C1 \\
Glutamate & Glutamate receptor ionotropic, kainate 3 & GRIK3 \\
Melatonin & Melatonin receptor 1A & MTNR1A \\
Neuropeptide & Brain-derived neurotrophic factor & BDNF \\
\hline
\end{tabular}

${ }^{\text {a }}$ Based on studies showing an association with alcohol withdrawal delirium [29].

Second, in studies seeking to associate delirium with biochemical markers in the blood, both delirium and the marker of interest could be influenced by the causal combination of predisposing and precipitating factors. Additionally, the moment in time over the 24 -hour observation period plays a confusing role because the symptoms of delirium as well as concentrations of many substances of interest fluctuate during the day. A concrete example of this problem is the study of diurnal secreted cortisol and delirium [31]. The level of cortisol is influenced by the predisposing factors of age and cognitive impairment, but it is affected even more by the stressful precipitating factor of delirium. In contrast with the biochemical markers, variations in a gene are fixed variables. For example, possible associations between delirium and a variation in a candidate gene involved in cortisol metabolism are not influenced by the factors causing delirium. Finding a genetic association is a clue that the gene product plays some role in the pathophysiological mechanism, but further research is needed to elucidate the gene's exact role.

The second aim of genetic research is to identify patients potentially at high risk for a certain disease [9]. The identification of genetic risk factors for delirium would permit individual patients prone to develop delirium to be recognized in advance. In this way, delirium would be missed less frequently. Preventive interventions could then be undertaken, and patients could be treated at an early stage. Moreover, the identification of genetic variations also provides an approach for adjusting pharmacotherapy at the individual level. Since delirium is a multifactorial disorder, the genetic make-up alone is likely insufficient to identify high-risk patients. Most probably, the classic major risk factors for delirium (e.g. advanced age and cognitive impairment) might be more predictive of delirium than genetic factors.

\section{Challenges of Genetic Research in Delirium}

The classic genetic research designs, such as family and twin studies, cannot be applied within the search for the heritability of delirium due to the reasons explained previously. Population-based gene studies can be useful to learn more about the association between genetic factors and delirium. A cross-sectional study design is the quickest method to find such an association, since phenotype and genotype are determined at the same time in a population of older people. Nonetheless, classifying the delirium phenotype adequately is impossible in this type of study. First, experienced clinicians can only properly diagnose delirium at the moment when the symptoms are present. Second, patients and their relatives are often unaware of whether they have ever experienced delirium. Therefore, a prospective study is the only design in which the diagnosis can be made adequately. 
An additional challenge for genetic studies is the inclusion of delirious patients. A sample size of at least hundreds of cases is required to provide sufficient power to discover a gene with a small effect [32]. Choosing an elderly hospital population with a high risk of delirium prevents the time-consuming and costly screening process that typically identifies too many controls without delirium. Unfortunately, these patients at risk for delirium often have an impaired capacity to provide consent due to pre-existing cognitive impairment, severe illness and the nature of delirium itself [33]. Current research regulations are stringent and may impede the collection of large populations of patients with delirium. In genetic research in elderly patients with dementia, the development of a consensus statement stimulated ethically acceptable research in this field [34]. Considering the similarities between dementia and delirium, genetic research in the field of delirium could also benefit by incorporating this statement.

A major pitfall in the comparison of genetic variations between cases and controls is that such a comparison could result in spurious associations if the controls are not completely matched to the cases with respect to factors influenced by an individual's genetic composition. If an association between a genetic factor and delirium is detected, it could in theory be related to the association of the genetic factor with cognitive impairment, since delirious patients are significantly more often cognitively impaired. Consequently, taking into account the influence of pre-existing cognitive impairment is an important issue in genetic studies on delirium in the elderly. Adequately diagnosing cognitive impairment during delirium poses a different problem, however, since temporary cognitive impairment is often one of the features of delirium itself. Assessing pre-existent cognitive function during delirium is only possible by interviewing a close relative. Instruments like the Informant Questionnaire on Cognitive Decline short form have been developed for this purpose, but they are not as reliable in assessing the patient's pre-existing cognitive status as tests of cognitive function administered before delirium [35].

Another challenge for successful genetic testing in delirium is the existence of many different hypotheses regarding the pathophysiology of the disease, because these hypotheses result in an abundance of available candidate genes with many possible SNPs. This introduces the risk of finding an association only by chance (i.e. a type $1 \mathrm{er}-$ ror). Correction for multiple tests will partially prevent false associations, but it simultaneously introduces the risk of missing interesting associations. Validation of as- sociations in an independent cohort offers extra certainty that a possible finding is not spurious. If the association is not replicated in a second cohort, however, heterogeneity couldbe responsiblefor the discrepancy. Although heterogeneity is not a unique difficulty for delirium studies, it is certainly larger in this phenotype due to problems in making an objective diagnosis as well as the underlying predisposing and precipitating factors. Consequently, validation under ideal conditions has to be performed in several hundreds of cases and control patients from different cohorts.

\section{Current Findings in Delirium Research}

Studies on genetics in delirium in elderly patients are scarce [36-42]. In addition to the obstacles discussed in this paper, the relative genetic ignorance of clinicians interested in delirium and geneticists' lack of knowledge of this syndrome may contribute to this scarcity. Up until now, 7 genetic studies on delirium have been published, all of them quite recently (table 2). Five of these studies examined the association of delirium with the apolipoprotein $\varepsilon 4$ allele [36, 38-41]. Given that (1) cognitive impairment is a strong risk factor for delirium and (2) cognitive impairment or dementia is associated with the apolipoprotein $\varepsilon 4$ allele, a link between delirium and the apolipoprotein $\varepsilon 4$ allele could be expected. The major role of APOE is to regulate cholesterol metabolism. In addition, it has been suggested that APOE in the central nervous system plays a role in neural plasticity, the repair of damaged neurons, as a neurotrophic factor, and in neural transmission [43]. The 3 different alleles $(\varepsilon 2, \varepsilon 3$ or $\varepsilon 4)$ are translated into the different apolipoproteins E (APOE2, APOE3 or APOE4), which vary in functionality and catabolism rates (the APOE4 being catabolized faster) [43, 44]. In animal studies it was shown that the APOE $\varepsilon 4$ genotype is associated with increased inflammation [45]. Additionally, the APOE $\varepsilon 4$ genotype reduces the cholinergic activity in the brain $[17,46]$. These theories of reduced cholinergic activity and the proinflammatory state are not mutually exclusive, since there is interaction between these systems. Animal studies have demonstrated that cytokines can cause a reduction in the acetylcholinergic pathways [47]. All of the above mechanisms could play a role in the enlarged risk on delirium in APOE $\varepsilon 4$ carriers.

Still, 4 of the 5 studies did not confirm this hypothesis, although 2 observed an association between the apolipoprotein $\varepsilon 4$ allele and a longer duration of delirium [36, 
Table 2. Genetic studies in delirium in the elderly

\begin{tabular}{|c|c|c|c|c|c|c|}
\hline Study & Study population & $\begin{array}{l}\text { Definition of } \\
\text { delirium }\end{array}$ & $\begin{array}{l}\text { Total } \\
\text { population }\end{array}$ & $\begin{array}{l}\text { Delirium } \\
\%\end{array}$ & $\begin{array}{l}\text { Candidate } \\
\text { gene }\end{array}$ & Association \\
\hline Adamis et al. [40], 2007 & elderly medical & CAM & 116 & 34 & APOE & - \\
\hline Ely et al. [36], 2007 & intensive care & CAM-ICU & 53 & 89 & APOE & - \\
\hline Leung et al. [39], 2007 & noncardiac surgery & CAM & 190 & 15 & APOE & + \\
\hline Tagarakis et al. [38], 2007 & cardiac surgery & DRS & 137 & 33 & APOE & - \\
\hline Tagarakis et al. [37], 2007 & cardiac surgery & DRS & 137 & 33 & SOAT-1 & - \\
\hline Van Munster et al. [41], 2007 & elderly medical & CAM & 264 & 35 & APOE & - \\
\hline \multirow[t]{3}{*}{ Van Munster et al. [42], 2009} & elderly medical & CAM & 720 & 37 & DRD2 & + \\
\hline & noncardiac surgery & & & & DRD3 & - \\
\hline & & & & & SLC6A & + \\
\hline
\end{tabular}

40]. The largest study population contains 264 patients, a number that is still far too small for a candidate gene study. Moreover, most of the trials did not take into account the important association existing between the apolipoprotein $\varepsilon 4$ allele and pre-existing cognitive impairment. Due to these limitations, we cannot definitely conclude that there is indeed no association between the apolipoprotein $\varepsilon 4$ allele and delirium.

The genetics of delirium due to alcohol withdrawal have been investigated more frequently, although these studies have also been conducted primarily with small numbers of patients [29]. Positive associations with alcohol withdrawal delirium were found for 3 different candidate genes involved in dopamine transmission: 1 gene involved in the glutamate pathway, 1 neuropeptide gene and 1 cannabinoid gene. Variations in 2 candidate genes involved in the dopamine transmission (the dopamine receptor D3 and the dopamine transporter) were validated in independent study populations. These analyses suggest that dopaminergic neurotransmission may play an important role in alcohol withdrawal delirium [29]. The findings in the DRD3 gene were not confirmed in an elderly population with delirium [42]. In the SLC6A3 gene, the variable number of tandem repeats was associated with alcohol withdrawal delirium. Although this variation was not confirmed in elderly patients with delirium, 2 other SNPs, rs393795 and rs1042098, in this gene were associated with delirium. Up to now, these polymorphisms have never been described in relation to any disease. One polymorphism in the DRD2 gene (rs6276) was associated with delirium after adjustment for the most important risk factors. The noncoding rs6276 SNP has been described in association with various alcohol-related phenotypes [48]. In conclusion, a protective effect for delirium in the elderly was found for 2 different genes involved in dopamine metabolism. The hypothesis of dopaminergic hyperactivity in delirium would be supported by these findings if the variations reduced the dopaminergic activity in the brain by, for example, changed availability or action of dopamine receptors or reduced attachment to these receptors $[49,50]$. Although the variations were all nonfunctional SNPs, they could be in linkage equilibrium with functional SNPs that do influence the dopaminergic activity. As this is yet unknown, it is speculative to state that these findings support the hypothesis of delirium being caused by enhanced dopaminergic activity in the brain.

\section{Future of Genetics in Delirium Research}

In this review, we have elucidated a possible role for genetics in delirium. The genetics of delirium are still in their infancy, but some progress is being made. Although studies on the genetics of delirium are hampered by many issues, we strongly urge the continuation of this type of research; the results are valuable tools for elucidating the pathophysiology of the disease. The fact that the investigation of delirium is relatively new in the field of genetic research allows us to learn from mistakes already made in genetic studies in other psychiatric disorders. For instance, many underpowered association studies in those psychiatric disorders were published but could not be replicated in validation trials [51]. Well-designed genetic association studies in delirium must utilize sufficient numbers of patients, proper classification of cases and controls, and adjustment for factors that could be falsely responsible for possible genetic associations. Moreover, 
the validation of new results in independent study cohorts is essential.

Currently, new cohort studies on delirium have started to collect DNA along with clinical parameters. Because of the small size of known delirium cohorts, international cooperation is warranted to perform candidate gene studies on a larger scale to permit any further progress. A meta-analysis of different study cohorts could provide an alternative tool to examine the data from these separate studies jointly. Even larger populations are needed to perform genome-wide association studies, which could generate new hypotheses about the pathophysiol- ogy of the disease [52]. If we continue on this road, we may in a few years understand more about the pathophysiology of delirium. Based on these findings, we may even develop improved markers or treatments for this population of vulnerable patients.

\section{Acknowledgements}

The authors wish to thank Hannah E. van Oosten and Annemarieke de Jonghe for critically reading the manuscript.

\section{References}

$\checkmark 1$ Michaud L, Bula C, Berney A, Camus V, Voellinger R, Stiefel F, Burnand B: Delirium: guidelines for general hospitals. J Psychosom Res 2007;62:371-383.

$\checkmark 2$ Inouye SK: Delirium in older persons. $\mathrm{N}$ Engl J Med 2006;354:1157-1165.

$\checkmark 3$ Maclullich AM, Ferguson KJ, Miller T, de Rooij SE, Cunningham C: Unravelling the pathophysiology of delirium: a focus on the role of aberrant stress responses. J Psychosom Res 2008;65:229-238.

4 Gunther ML, Morandi A, Ely EW: Pathophysiology of delirium in the intensive care unit. Crit Care Clin 2008;24:45-65.

$\checkmark 5$ Marcantonio ER, Rudolph JL, Culley D, Crosby G, Alsop D, Inouye SK: Serum biomarkers for delirium. J Gerontol A Biol Sci Med Sci 2006;61:1281-1286.

6 Blacker D, Lovestone S: Genetics and dementia nosology. J Geriatr Psychiatry Neuro 2006;19:186-191.

7 Beck AT: The evolution of the cognitive model of depression and its neurobiological correlates. Am J Psychiatry 2008;165:969977.

$\checkmark 8$ Maier W: Common risk genes for affective and schizophrenic psychoses. Eur Arch Psychiatry Clin Neurosci 2008;258(suppl 2):3740.

-9 Zuchner S, Roberts ST, Speer MC, Beckham JC: Update on psychiatric genetics. Genet Med 2007;9:332-340.

10 American Psychiatric Association: Diagnostic and Statistical Manual of Mental Disorders: DSM IV-TR. Washington, American Psychiatric Association, 2000.

-11 Meagher DJ, Maclullich AM, Laurila JV: Defining delirium for the International Classification of Diseases, 11th Revision. J Psychosom Res 2008;65:207-214.

-12 Korevaar JC, van Munster BC, de Rooij SE: Risk factors for delirium in acutely admitted elderly patients: a prospective cohort study. BMC Geriatr 2005;5:6.
3 Rockwood K, Cosway S, Carver D, Jarrett P, Stadnyk K, Fisk J: The risk of dementia and death after delirium. Age Ageing 1999;28: 551-556.

14 Van der Mast RC, Huyse FJ, Rosier PF: Guideline 'Delirium'. Ned Tijdschr Geneeskd 2005;149:1027-1032.

15 Inouye SK, Foreman MD, Mion LC, Katz KH, Cooney LM, Jr: Nurses' recognition of delirium and its symptoms: comparison of nurse and researcher ratings. Arch Intern Med 2001;161:2467-2473.

16 Meagher DJ, Trzepacz PT: Motoric subtypes of delirium. Semin Clin Neuropsychiatry 2000;5:75-85.

17 Trzepacz PT: Is there a final common neural pathway in delirium? Focus on acetylcholine and dopamine. Semin Clin Neuropsychiatry 2000;5:132-148.

18 Maldonado JR: Pathoetiological model of delirium: a comprehensive understanding of the neurobiology of delirium and an evidence-based approach to prevention and treatment. Crit Care Clin 2008;24:789-856.

-19 Alagiakrishnan K, Wiens CA: An approach to drug-induced delirium in the elderly. Postgrad Med J 2004;80:388-393.

20 Reichenberg A, Yirmiya R, Schuld A, Kraus T, Haack M, Morag A, Pollmacher T: Cytokine-associated emotional and cognitive disturbances in humans. Arch Gen Psychiatry 2001;58:445-452.

21 De Rooij SE, van Munster BC, Korevaar JC, Levi M: Cytokines and acute phase response in delirium. J Psychosom Res 2007;62:521525.

22 Van Munster BC, Korevaar JC, Zwinderman AH, Levi M, Wiersinga WJ, de Rooij SE: Time course of cytokines during delirium in elderly patients with hip fractures. J Am Geriatr Soc 2008;56:1704-1709.

23 Lewis MC, Barnett SR: Postoperative delirium: the tryptophan dyregulation model. Med Hypotheses 2004;63:402-406.
24 Shigeta H, Yasui A, Nimura Y, Machida N, Kageyama M, Miura M, Menjo M, Ikeda K: Postoperative delirium and melatonin levels in elderly patients. Am J Surg 2001;182:449454.

25 Inouye SK, Charpentier PA: Precipitating factors for delirium in hospitalized elderly persons: predictive model and interrelationship with baseline vulnerability. JAMA 1996; 275:852-857.

26 Bacanu SA, Devlin B, Chowdari KV, DeKosky ST, Nimgaonkar VL, Sweet RA: Heritability of psychosis in Alzheimer disease. Am J Geriatr Psychiatry 2005;13:624-627.

$>27$ Kennedy JL, Farrer LA, Andreasen NC, Mayeux R, St George-Hyslop P: The genetics of adult-onset neuropsychiatric disease: complexities and conundra? Science 2003; 302:822-826.

28 Baudoin C, Cohen-Solal ME, Beaudreuil J, De Vernejoul MC: Genetic and environmental factors affect bone density variances of families of men and women with osteoporosis. J Clin Endocrinol Metab 2002;87:20532059.

29 Van Munster BC, Korevaar JC, de Rooij SE, Levi M, Zwinderman AH: Genetic polymorphisms related to delirium tremens: a systematic review. Alcohol Clin Exp Res 2007; 31:177-184.

30 Abdolmaleky HM, Thiagalingam S, Wilcox $\mathrm{M}$ : Genetics and epigenetics in major psychiatric disorders: dilemmas, achievements, applications, and future scope. Am J Pharmacogenomics 2005;5:149-160.

$\checkmark 31$ Olsson T: Activity in the hypothalamic-pituitary-adrenal axis and delirium. Dement Geriatr Cogn Disord 1999;10:345-349.

-32 Edwards BJ, Haynes C, Levenstien MA, Finch SJ, Gordon D: Power and sample size calculations in the presence of phenotype errors for case/control genetic association studies. BMC Genet 2005;6:18.

33 Holt R, Siddiqi N, Young J: The ethics of consent in delirium studies. J Psychosom Res 2008;65:283-287. 
34 Olde Rikkert MG, van der Vorm A, Burns A, Dekkers W, Robert P, Sartorius N, Selmes J, Stoppe G, Vernooij-Dassen M, Waldemar G: Consensus statement on genetic research in dementia. Am J Alzheimers Dis Other Demen 2008;23:262-266.

>35 Jorm AF: A short form of the Informant Questionnaire on Cognitive Decline in the Elderly (IQCODE): development and crossvalidation. Psychol Med 1994;24:145-153.

>36 Ely EW, Girard TD, Shintani AK, Jackson JC, Gordon SM, Thomason JW, Pun BT, Canonico AE, Light RW, Pandharipande P, Laskowitz DT: Apolipoprotein E4 polymorphism as a genetic predisposition to delirium in critically ill patients. Crit Care Med 2007; 35:112-117.

\37 Tagarakis GI, Tsolaki-Tagaraki F, Tsolaki M, Diegeler A, Kazis D, Rouska E, Papassotiropoulos A: The role of SOAT-1 polymorphisms in cognitive decline and delirium after bypass heart surgery. Clin Res Cardiol 2007;96:600-603.

-38 Tagarakis GI, Tsolaki-Tagaraki F, Tsolaki M, Diegeler A, Tsilimingas NB, Papassotiropoulos $\mathrm{A}$ : The role of apolipoprotein $\mathrm{E}$ in cognitive decline and delirium after bypass heart operations. Am J Alzheimers Dis Other Demen 2007;22:223-228.

-39 Leung JM, Sands LP, Wang Y, Poon A, Kwok PY, Kane JP, Pullinger CR: Apolipoprotein E e4 allele increases the risk of early postoperative delirium in older patients undergoing noncardiac surgery. Anesthesiology 2007; 107:406-411.
40 Adamis D, Treloar A, Martin FC, Gregson N, Hamilton G, Macdonald AJ: APOE and cytokines as biological markers for recovery of prevalent delirium in elderly medical inpatients. Int J Geriatr Psychiatry 2007;22:688694.

41 Van Munster BC, Korevaar JC, de Rooij SE, Levi M, Zwinderman AH: The association between delirium and the apolipoprotein $\mathrm{E}$ $\varepsilon 4$ allele in the elderly. Psychiatr Genet 2007; 17:261-266.

42 Van Munster BC, Yazdanpanah M, Tanck MW, de Rooij SE, van de Giessen E, Sijbrands EJ, Zwinderman AH, Korevaar JC: Genetic polymorphisms in the DRD2, DRD3, and SLC6A3 gene in elderly patients with delirium. Am J Med Genet B Neuropsychiatr Genet 2009; Mar 23, Epub ahead of print.

43 Adamis D, Macdonald AJ: A review of the association of apolipoprotein E and delirium; in Penfield LR, Nelson RT (eds): Apoprotein Research. Hauppauge, Nova Science Publishers, 2009.

44 Siest G, Pillot T, Regis-Bailly A, LeiningerMuller B, Steinmetz J, Galteau MM, Visvikis S: Apolipoprotein E: an important gene and protein to follow in laboratory medicine. Clin Chem 1995;41:1068-1086.

45 Vasto S, Candore G, Listi F, Balistreri CR, Colonna-Romano G, Malavolta M, Lio D, Nuzzo D, Mocchegiani E, Di BD, Caruso C: Inflammation, genes and zinc in Alzheimer's disease. Brain Res Rev 2008;58:96105.
46 Allen SJ, MacGowan SH, Tyler S, Wilcock GK, Robertson AG, Holden PH, Smith SK, Dawbarn D: Reduced cholinergic function in normal and Alzheimer's disease brain is associated with apolipoprotein E4 genotype. Neurosci Lett 1997;239:33-36.

47 Willard LB, Hauss-Wegrzyniak B, Wenk GL: Pathological and biochemical consequences of acute and chronic neuroinflammation within the basal forebrain cholinergic system of rats. Neuroscience 1999;88:193-200.

48 Lucht M, Barnow S, Schroeder W, Grabe HJ, Rosskopf D, Brummer C, John U, Freyberger $\mathrm{HJ}$, Herrmann FH: Alcohol consumption is associated with an interaction between DRD2 exon $8 \mathrm{~A} / \mathrm{A}$ genotype and self-directedness in males. Neuropsychobiology 2007; $56: 24-31$.

49 Nikolaus S, Antke C, Kley K, Poeppel TD, Hautzel H, Schmidt D, Muller HW: Investigating the dopaminergic synapse in vivo. I. Molecular imaging studies in humans. Rev Neurosci 2007;18:439-472.

50 Van Dyck CH, Malison RT, Jacobsen LK, Seibyl JP, Staley JK, Laruelle M, Baldwin RM, Innis RB, Gelernter J: Increased dopamine transporter availability associated with the 9-repeat allele of the SLC6A3 gene. J Nucl Med 2005;46:745-751.

-51 Stoltenberg SF, Burmeister M: Recent progress in psychiatric genetics - some hope but no hype. Hum Mol Genet 2000;9:927-935.

52 Lango H, Weedon MN: What will whole genome searches for susceptibility genes for common complex disease offer to clinical practice? J Intern Med 2008;263:16-27. 